\title{
Traceurs isotopiques : sources et processus
}

\author{
A.M. Fouillac ${ }^{1}$, A. Cocherie ${ }^{1}$, J.P. Girard' ${ }^{1}$, C. Guerrot ${ }^{1}$, C. Innocent ${ }^{1}$, R. Millot ${ }^{1}$, \\ M. Motelica ${ }^{1}$, B. Sanjuan ${ }^{1}$ et D. Widory ${ }^{1}$ \\ 1 BRGM, 2, avenue Caude-Guillemin, BP 6009, 45060 Orléans Cedex - France \\ e-mail : am.fouillac@brgm.fr - a.cocherie@brgm.fr - jp.girard@brgm.fr - c.guerrot@brgm.fr - c.innocent@brgm.fr - r.millot@brgm.fr \\ m.motelica@brgm.fr - b.sanjuan@brgm.fr - d.widory@brgm.fr
}

Résumé - Des évolutions technologiques majeures ont eu lieu ces dernières années dans le domaine de la mesure isotopique. L'objectif de cet article est de montrer le potentiel de ces avancées appliquées aux sciences de la terre et de l'environnement.

Il s'agit, d'une part, de la technologie des spectromètres de masse à flux continu, dont la fiabilité est maintenant acquise à des précisions semblables à celles des spectromètres de masse à source gazeuse et double introduction, mais sur des échantillons beaucoup plus petits et avec une productivité largement supérieure. D'autre part, cet article expose le développement des ICP-MS-MC (spectromètre de masse à multicollection et source à plasma induit). Ces instruments permettent de réaliser des mesures isotopiques dont la précision et la justesse rivalisent avec celles obtenues en TIMS (spectromètre de masse à thermoionisation), mais sur une panoplie d'éléments chimiques beaucoup plus large, à des concentrations très basses, et avec une rapidité bien supérieure ( $\mathrm{Cr}, \mathrm{Zr}, \mathrm{Mo}, \mathrm{Cu}, \mathrm{Zn}, \mathrm{Fe}, \mathrm{Mg}, \mathrm{Cd}, \mathrm{Li}, \mathrm{B}, \mathrm{Hf}, \mathrm{Se}, \mathrm{Pb})$.

Ces avancées permettent un accès plus facile aux techniques isotopiques, en termes de productivité et donc de coût, en termes de taille d'échantillons, et enfin, d'investigation grâce à l'accès à de nouveaux systèmes isotopiques. Ceci favorise la mise en œuvre d'études basées sur plusieurs traceurs isotopiques dont la complémentarité renforce la puissance.

Ces nouvelles perspectives seront exposées au travers d'exemples pris dans le domaine de la géothermie, du stockage de déchets, de la migration de fluides et du transport aérien de matière particulaire en milieu urbain.

Abstract - Isotopic Tracers : Sources and Processes - Major technological advances have taken place in the past few years in the field of isotopic measurements. The goal of the present paper is to illustrate the potential unveiled by such advances in their application to geosciences and environmental issues.

One significant improvement in analytical performance was brought by continuous flow mass spectrometry (CF-IRMS), a technology which demonstrated its capability to provide isotopic measurements at a level of accuracy comparable to conventional dual-inlet gas-source mass spectrometry. The advantage of CF-IRMS over conventional IRMS however consists in higher sensitivity and greater productivity.

A second major analytical advance lies in the development of the multi-collection ICP-MS (MC-ICP-MS, multi-collection inductively coupled plasma mass spectrometer). This equipment permits to measure isotopic ratios of a wide variety of chemical elements, with a precision comparable, or better, than that of thermoionisation mass spectrometers. In addition, MC-ICP-MS offers great sensitivity, allowing isotopic measurements at extremely low concentration levels, high sample throughput, and provides access to 
elements that are notoriously difficult to analyze or cannot be analyzed by $\mathrm{TIMS}(\mathrm{Cr}, \mathrm{Zr}, \mathrm{Mo}, \mathrm{Cu}, \mathrm{Zn}, \mathrm{Fe}$, $\mathrm{Mg}, \mathrm{Cd}, \mathrm{Li}, \mathrm{B}, \mathrm{Hf}$, Se, $\mathrm{Pb}$ ).

Thank to these instrumental advances, investigators benefit from an easier and faster access to isotopic measurements, at significantly lower costs. The possibility to analyze samples of much smaller size, and elements which isotopic systems had not been explored yet, opens up new areas of research and favors the use of multi-isotope approaches in isotopic studies. Some of these new perspectives are illustrated through a few on-going studies carried out at BRGM, in the fields of geothermal energy, waste storage and fluid migration, and atmospheric particles in urban areas.

\section{INTRODUCTION}

Dans le contexte actuel, en réponse à la demande sociétale et aux préoccupations des pouvoirs publics, les axes de recherche du BRGM (Bureau de recherche géologique et minière) portent sur le management de la ressource minérale, de la ressource en eau, de l'énergie et sur le management des impacts environnementaux concernant la qualité de l'eau, de l'air et du sol. Ceci inclut l'étude des stockages de déchets industriels et nucléaires, le suivi des activités minières et industrielles.

Globalement, ces axes de recherche concernent l'interaction entre les trois compartiments air, eau et sol. Ceux-ci deviennent rapidement indissociables dans le cadre d'études environnementales nécessitant l'identification de pôles de mélanges et la compréhension des processus d'interaction mis en jeu. Dans ces études, il est classiquement fait appel, en première approche, aux méthodes de caractérisation physique et chimique des différentes phases pour résoudre le problème. Si la complexité de l'étude le nécessite, on a ensuite recours à l'utilisation de traceurs isotopiques.

Nous traiterons ici des mesures de rapports isotopiques d'éléments dans le milieu naturel et à des teneurs naturelles. Les grandes applications en géosciences sont la datation des roches et des événements géologiques, la géothermométrie isotopique et le traçage géochimique. C'est cette dernière utilisation qui sera principalement développée ici, sur quelques exemples, montrant l'utilisation actuelle et les nouvelles perspectives permises grâce à l'évolution technologique considérable de ces dernières années. Dans tous les cas, les études démontrent le potentiel de l'outil multi-isotopique, c'est-à-dire de l'utilisation de plusieurs traceurs isotopiques aux propriétés complémentaires.

\section{1 ÉVOLUTION TECHNOLOGIQUE DE L'OUTIL ISOTOPIQUE}

Trois types d'instruments de mesure isotopique sont maintenant prédominants en sciences de la terre et de l'environnement: la sonde ionique haute résolution (HR-SIMS), le spectromètre de masse à flux continu (CF-IRMS) et le spectromètre de masse à secteur magnétique, multicollection et source à plasma induit (ICP-MS-MC).

\subsection{Sonde ionique haute résolution}

La sonde ionique haute résolution permet la mesure in situ de rapports isotopiques avec une haute résolution spatiale. Le diamètre de la zone d'impact est de l'ordre de $10 \mu \mathrm{m}$. Ce potentiel de mesure in situ sur lame mince ou section polie d'échantillon est naturellement son principal atout. Pour de nombreux éléments, la précision atteinte permet maintenant de satisfaire de nombreux objectifs scientifiques ou industriels, bien qu'elle reste en deçà de la précision atteinte avec les techniques ex situ. La difficulté majeure demeure le besoin fondamental en matériaux de référence solides et homogènes en composition isotopique pour chaque élément et pour chaque type de matrice considéré.

\subsection{CF-IRMS : spectromètre de masse à flux continu}

La spectrométrie de masse à source gazeuse, en mode dynamique ou statique, permet la mesure de rapport isotopique d'éléments légers. Il y a une dizaine d'années, cette technique a été enrichie par le développement de la spectrométrie de masse à source gazeuse en flux continu. Les premières générations d'appareils péchaient par une précision analytique dégradée. Actuellement, le spectromètre de masse à flux continu permet de réaliser des mesures isotopiques dont la précision et la justesse rivalisent avec celles obtenues par le mode dynamique ou statique. De plus, cette nouvelle technologie présente également trois avantages énormes :

- la possibilité de travailler sur des volumes d'échantillon extrêmement faibles. En effet, le gain de sensibilité est d'un facteur pouvant atteindre 1000 par rapport à un spectromètre de masse dynamique ;

- la possibilité d'effectuer l'extraction en ligne des espèces ciblées à partir d'un échantillon contenant plusieurs autres espèces par pyrolyse et/ou oxydation, et chromatographie en phase gazeuse ;

- enfin, elle permet d'augmenter de façon très significative la productivité analytique par automatisation des différentes phases de préparation et d'analyse.

\subsection{ICP-MS-MC, spectromètre de masse à multicollection et source à plasma induit}

Depuis plus de 20 ans, la spectrométrie de masse à thermoionisation (TIMS) constitue la technologie de référence pour 
la mesure de rapports isotopiques à un degré de précision élevé. Cette suprématie est remise en cause par le développement d'une nouvelle génération d'ICP-MS, qui combine la souplesse et la rapidité d'ionisation d'une source ICP avec la précision d'un spectromètre de masse à multicollection. $\mathrm{Ce}$ sont les ICP-MS à multicollection et secteur magnétique (ICP-MS-MC). Ces instruments permettent de réaliser des mesures isotopiques dont la précision et la justesse rivalisent avec celle obtenues en TIMS mais sur une panoplie d'éléments chimiques beaucoup plus large, même à des concentrations très basses, et avec une rapidité bien supérieure. Cette avancée technologique offre donc une nette amélioration de la productivité et se révèle indispensable dans des domaines qui nécessitent le traitement d'un grand nombre de données analytiques, tels que l'environnement et de la qualité des eaux.

Des éléments difficiles à analyser en TIMS, en raison de leur caractère peu ionisable, tels que le $\mathrm{Cr}, \mathrm{Zr}, \mathrm{Mo}, \mathrm{Cu}, \mathrm{Zn}, \mathrm{Fe}$, $\mathrm{Mg}, \mathrm{Cd}$, etc. sont accessibles en ICP/MS-MC. D'autres éléments comme $\mathrm{Li}, \mathrm{B}, \mathrm{Hf}$, Se et $\mathrm{Pb}$, analysables en TIMS, peuvent être analysés en ICP-MS-MC beaucoup plus facilement et plus rapidement. Par ailleurs, le couplage à un système d'ablation laser approprié permet d'envisager, dans certains cas, l'analyse directe sur minéraux, à la manière de ce qui est fait avec les sondes ioniques mais avec une résolution spatiale inférieure. Enfin, la potentialité de coupler ces appareils avec des systèmes de séparation, tels que les systèmes chromatographiques, laisse la voie ouverte à de nombreuses avancées scientifiques dans le domaine de la caractérisation isotopique d'espèces chimiques individuelles, organiques ou minérales.

L'ensemble de la communauté scientifique des géochimistes s'accorde à penser que cette technologie représente la voie du futur, dans le domaine de la géochimie des traces, ultra-traces et de la géochimie isotopique.

\subsection{Illustration des potentialités du ICP-MS-MC sur le cas du lithium}

Les développements prioritaires, qui ont été réalisés au $B R G M$ concernent le lithium, le plomb, le thorium, le bore, le cadmium et le fer. Parmi ceux-ci, une bonne illustration des potentialités du ICP-MS-MC est donnée par le travail analytique mené sur le lithium (Millot et al., 2004).

L'objectif était de développer une méthode analytique pour la mesure précise du rapport isotopique du lithium sur de faibles quantités, de l'ordre de 15 à 20 ng.

Pour cela, une étape préliminaire de purification chimique par chromatographie sur résine échangeuse d'ions est nécessaire et le protocole a été validé pour des matrices liquides et solides (eaux et roches). Le rendement de cette purification doit impérativement être de $100 \%$ afin d'éviter tout fractionnement isotopique au cours de cette étape. Lors de la mesure des isotopes du lithium par ICP-MS-MC, le biais de masse instrumental est corrigé par les mesures alternées de standard (L-SVEC) et d'échantillon, selon la méthode dite de standard- bracketing. La reproductibilité analytique que nous obtenons est de $\pm 0.5 \%$ o (2 écarts types) ; en cela, notre méthode analytique représente un net progrès en termes de reproductibilité par rapport aux autres laboratoires ICP-MS-MC.

La qualité du travail analytique réalisé sur cet instrument est illustrée par la figure 1. Dans cette figure sont reportés les résultats compilés de la littérature sur la mesure du $\delta^{7} \mathrm{Li}$ de l'eau de mer par TIMS et ICP-MS-MC. Les résultats obtenus par le $B R G M$ ont été réalisés sur un ICP-MS-MC de type Neptune (Thermo-Electron Corporation).

Plusieurs remarques ressortent de ce travail. Tout d'abord, le fait de considérer l'eau de mer comme un échantillon naturel de référence peut être discuté. En effet, lorsque l'on compare les analyses isotopiques de l'eau de mer, non seulement la reproductibilité de la mesure est prise en compte, mais également, la qualité de l'étape de purification (en termes de répétabilité), s'agissant d'un échantillon à forte matrice. D'autre part, on peut se poser la question quant à l'homogénéité isotopique en lithium de l'océan à l'échelle mondiale. En effet, plusieurs résultats de la littérature résultent d'analyses faites sur des échantillonnages d'eau de mer qui ne sont pas des matériaux de référence. C'est pourquoi, nous avons proposé que soit adoptée par la communauté scientifique l'eau de mer de référence IRMM BCR-403 comme standard pour les analyses isotopiques en lithium.

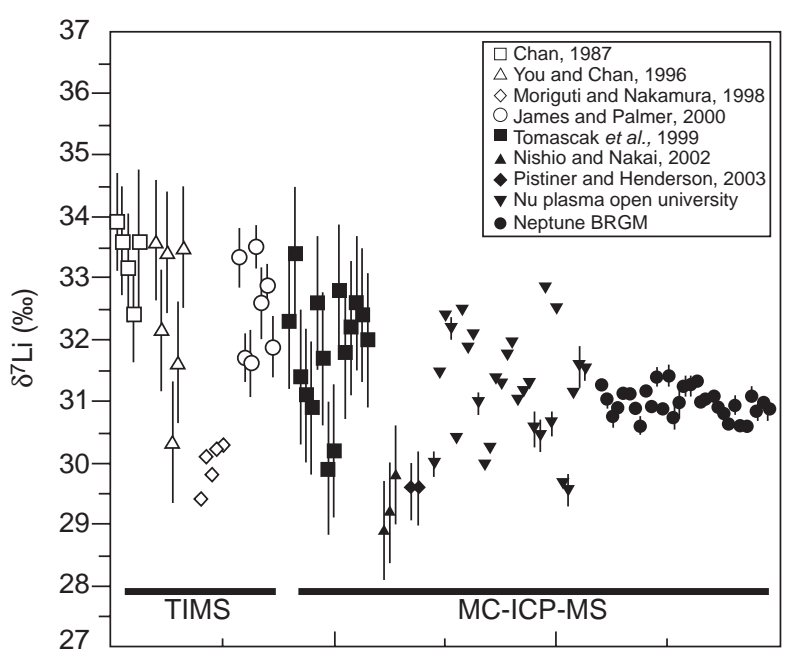

Figure publiée initialement dans Geostandards and Geoanalytical Research (2004, Vol. 28, ${ }^{\circ} 1$, pp. 153-159), reproduite avec la permission de l'Association scientifique pour la Géologie et ses applications (ASGA) détenteur des droits.

Figure 1

Comparaison de résultats obtenus sur l'eau de mer par différentes équipes en TIMS et ICP-MS-MC (Millot et al. 2004). Les résultats obtenus par le $B R G M$ ont été réalisés sur un ICP-MS-MC de type Neptune (Thermo-Electron Co.).

Comparison of sea-water composition obtained from different TIMS and MC-ICP-MS laboratories (Millot et al., 2004). BRGM results have been obtained with a ThermoElectron Co. Neptune MC-ICP-MS. 


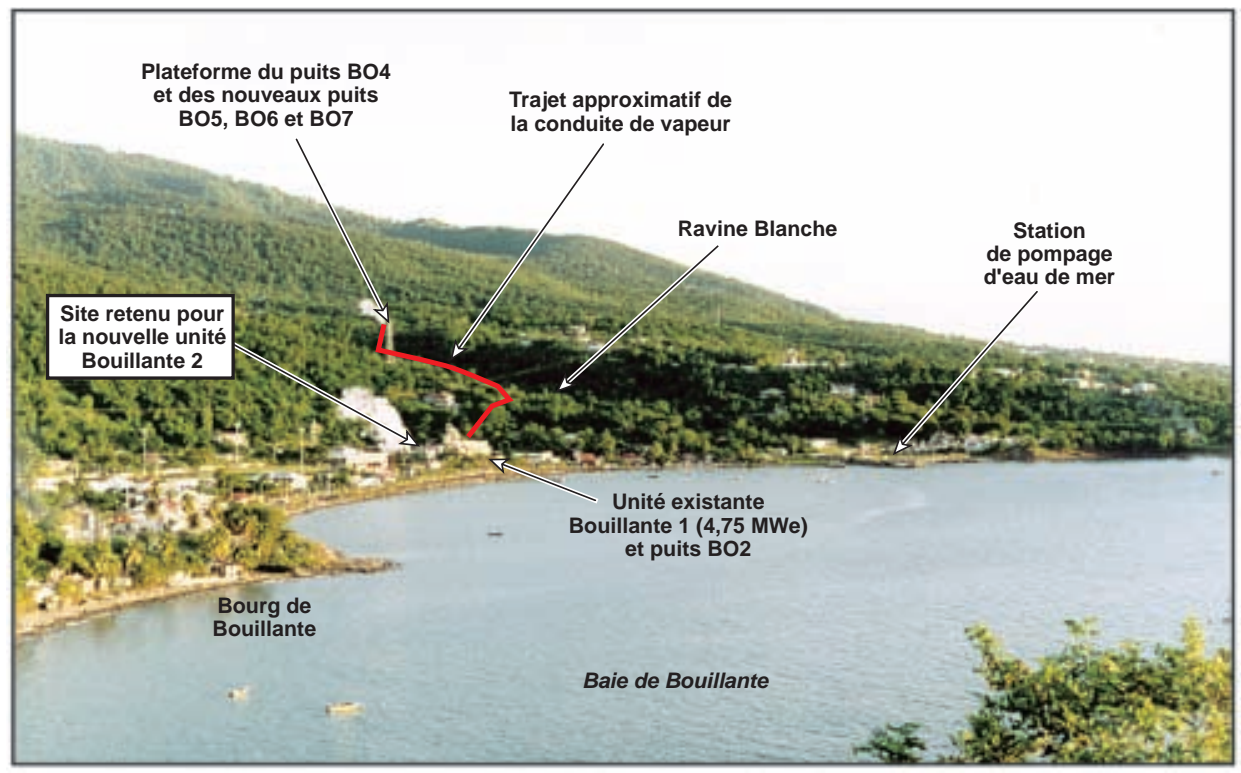

Figure 2

Site de la centrale géothermique de Bouillante, en Guadeloupe.

Setting of the Bouillante geothermal plant, Guadeloupe.

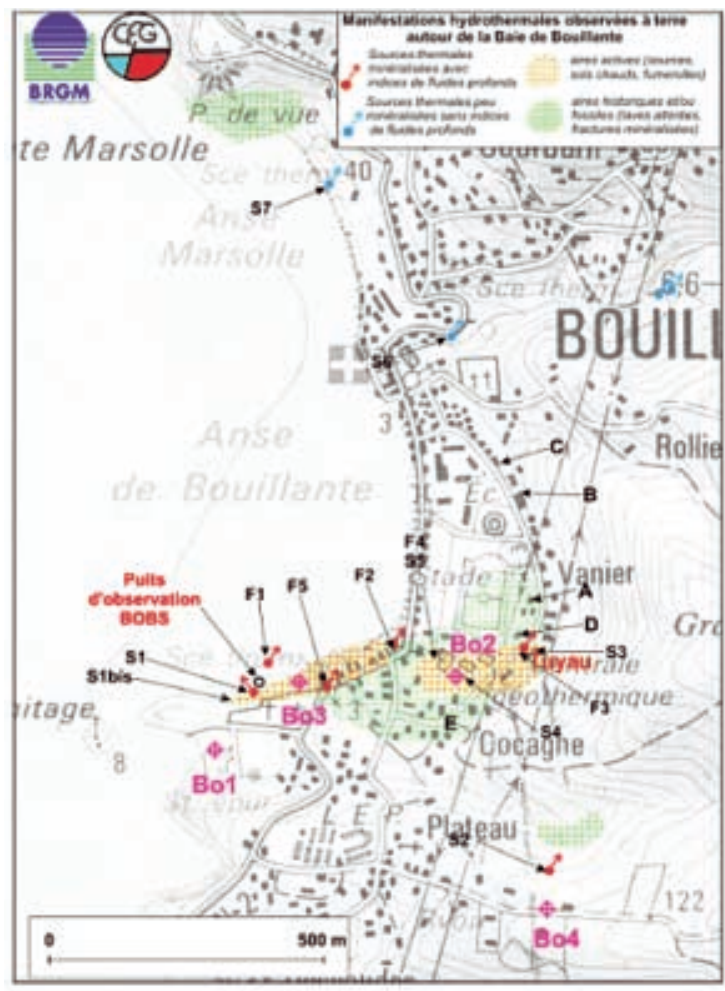

Figure 3

Carte des points de prélèvements utilisés pour la surveillance géochimique du site de la centrale de Bouillante, Guadeloupe. Map of the sampling spots used for the geochemical monitoring of the Bouillante geothermal plant, Guadeloupe.
Ce matériau de référence n'est pas certifié pour les mesures de rapports isotopiques mais pour l'analyse d'éléments traces. Toutefois, il s'agit d'un échantillonnage d'eau de mer représentatif, homogène et disponible auprès de l'IRMM (Institute of Reference Materials and Measurements). C'est sur ce standard d'eau de mer que nous avons déterminé une valeur moyenne de $\delta^{7} \mathrm{Li}=31.0 \% 0 \pm 0.5(2 \sigma, \mathrm{n}=31)$. Quoiqu'il en soit, la figure 1 illustre bien la qualité de notre mesure en termes de reproductibilité, comparé aux autres laboratoires TIMS et ICP-MS-MC.

Les premières applications de la mesure des $\delta^{7} \mathrm{Li}$ au $B R G M$ concernent le domaine de la géothermie.

\section{APPLICATIONS}

\section{1 Énergies renouvelables : la géothermie}

Dans un contexte mondial de consommation énergétique en forte augmentation, le développement d'énergies renouvelables s'intensifie. Dans les Antilles françaises, la géothermie haute enthalpie pourrait constituer une alternative économiquement viable à l'énergie actuelle. Le champ géothermique de Bouillante (Guadeloupe), exploité par la société Géothermie Bouillante, est en cours de développement. L'un des principaux objectifs des travaux de recherche menés par le $B R G M$ est d'accroître et de sécuriser la production d'électricité d'origine géothermique et d'identifier les meilleures méthodes d'exploration et quantification des ressources. Ceci implique, entre autres, une estimation de la température et du 
volume du réservoir profond. Par ailleurs, les problèmes liés à l'utilisation du fluide géothermal (risques de dépôts dans les canalisations et échangeurs, rejets contaminés en métaux ou métalloïdes) doivent également être pris en compte.

Tous ces travaux de recherche exigent non seulement la réalisation d'analyses chimiques des fluides, mais aussi, celle de leurs analyses isotopiques $\left(\delta \mathrm{D}\right.$ et $\delta^{18} \mathrm{O}, \delta^{7} \mathrm{Li}, \delta^{11} \mathrm{~B}$, ${ }^{87} \mathrm{Sr} /{ }^{86} \mathrm{Sr}, \delta^{13} \mathrm{C}$, etc.).

\subsubsection{Suivi et surveillance de l'environnement d'une centrale géothermique au cours de son exploitation (site de la centrale de Bovillante)}

L'étude en cours porte sur l'utilisation d'outils classiques de chimie (analyses des majeurs et des traces) et de géochimie isotopique (analyses $\delta \mathrm{D}$ et $\delta^{18} \mathrm{O}$ des fluides) appliqués à des eaux thermales superficielles et profondes pour surveiller l'impact de l'exploitation de la centrale géothermale sur son environnement immédiat, notamment sur les aquifères superficiels, voire sur le réservoir profond (Sanjuan et al., 2000 ; 2001). Le suivi et la surveillance du site sont réalisés à partir de plusieurs points de prélèvement (fig. 2 et 3 ) :

- le puits d'observation BOBS situé en bord de mer est suivi depuis 1999. C'est un petit forage de $7 \mathrm{~m}$, qui capte un fluide géothermal profond du type de celui des forages $\mathrm{BO} 2$ et $\mathrm{BO} 4$ ayant subi une étape de vaporisation ;
- la source Tuyau (S3), suivie depuis 1996. Elle se situe juste derrière la centrale géothermique ;

- plusieurs sources thermales situées autour de la centrale ainsi que des fumerolles.

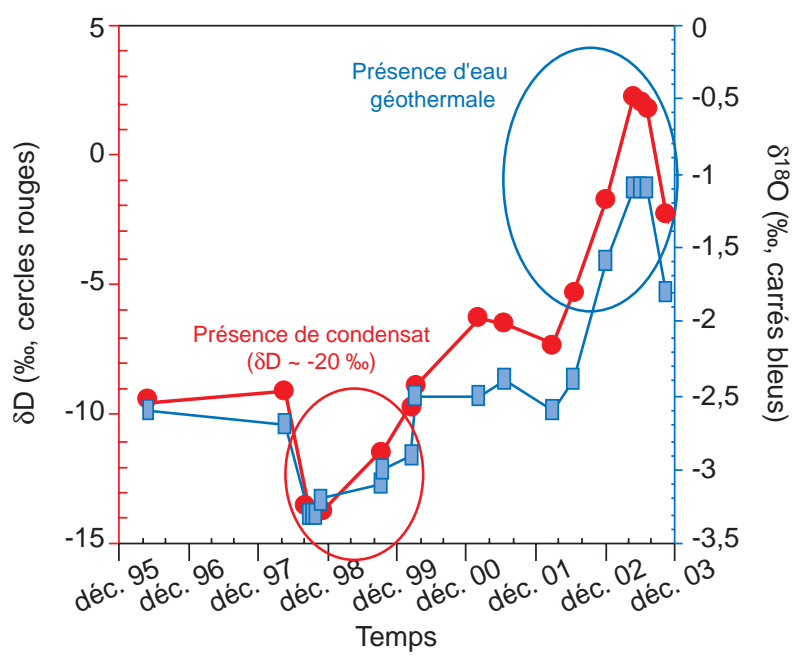

Figure 4

Évolution des compositions isotopiques du fluide de la source Tuyau (Bouillante, Guadeloupe).

Isotopic composition changes of the Tuyau spring fluids (Bouillante, Guadeloupe).

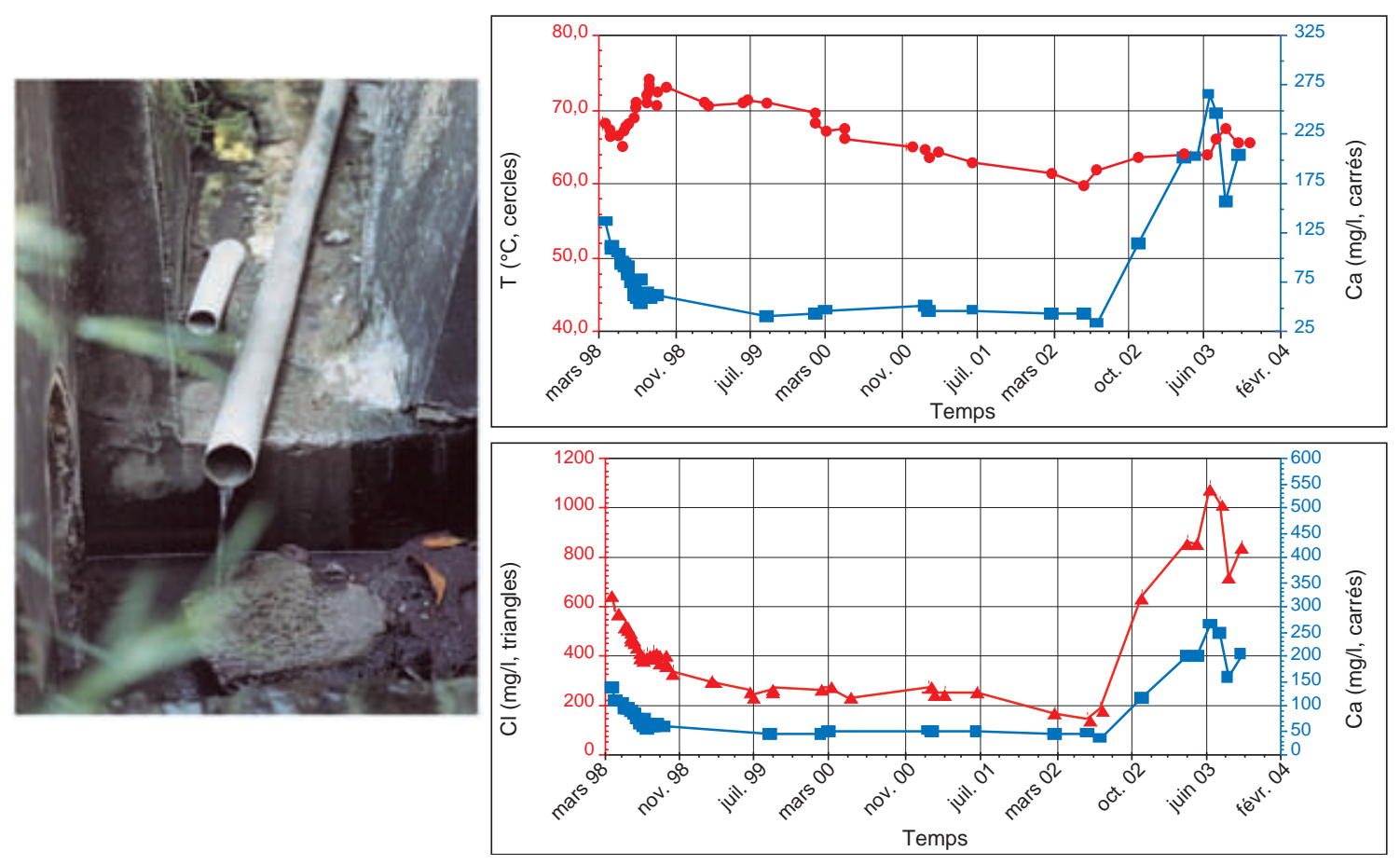

Figure 5

Évolution de la température et de la composition chimique du fluide de la source Tuyau (Bouillante, Guadeloupe).

Temperature and chemical composition changes of the Tuyau spring fluids (Bouillante, Guadeloupe). 


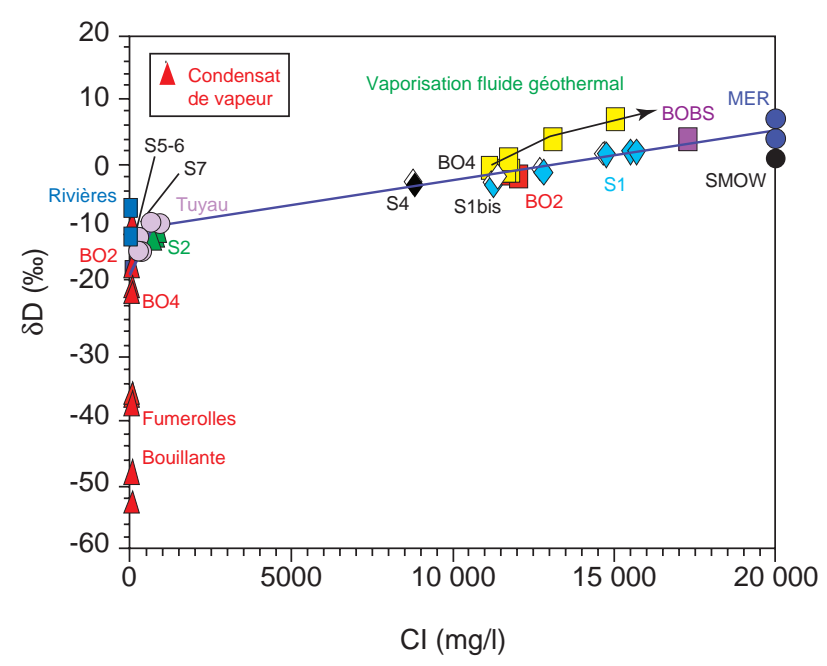

Figure 6

Compositions en chlorures et $\delta \mathrm{D}$ des fluides thermaux de la région de Bouillante.

Chloride compositions and $\delta D$ of the Bouillante area thermal fluids.

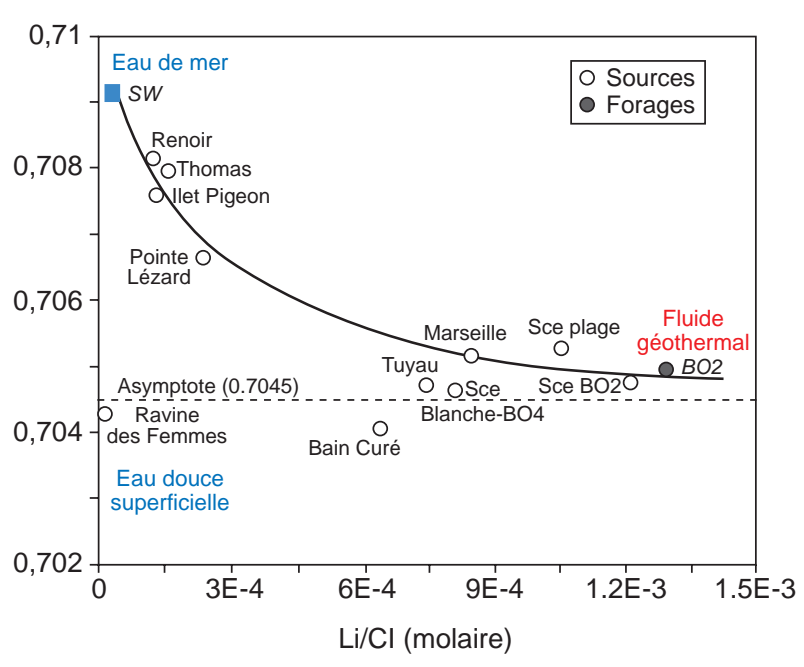

Figure 7

Variation des compositions isotopiques du strontium des fluides de la région de Bouillante.

Changes in strontium isotopic composition of the Tuyau spring fluids, Bouillante.
En 1996, les analyses chimiques et isotopiques de l'eau de la source Tuyau ont indiqué que celle-ci était principalement constituée d'eau douce superficielle, d'un peu d'eau géothermale profonde et éventuellement d'eau de mer. La diminution de la salinité (de 2 à $1 \mathrm{~g} / \mathrm{l}$ ), des concentrations en chlorure et calcium en solution, l'évolution de la signature en isotopes stables de l'eau ainsi que l'augmentation de la température (de 60 à $74^{\circ} \mathrm{C}$ ), observées pendant une grande partie du suivi géochimique de cette source (mai 1996-octobre 1998), ont suggéré une contribution de plus en plus importante d'une phase de condensat de vapeur en surface (fig. : 4 et 6). Cette évolution a été probablement causée par l'exploitation de la centrale géothermique. À partir d'octobre 1998, la température à l'émergence a eu tendance à diminuer légèrement tandis que la composition chimique du fluide s'est plutôt stabilisée. Les valeurs de deutérium et d'oxygène-18 mesurées ont indiqué une proportion d'eau douce superficielle de plus en plus importante dans le fluide de cette source et une diminution de la contribution du pôle de condensat de vapeur, avec une quasi-disparition de ce pôle, en mars 2001. À la mi-2002, depuis l'arrêt du forage BO2, relativement superficiel (330 $\mathrm{m}$ de profondeur), qui alimentait la centrale, la légère augmentation de salinité et des concentrations en chlorure et en calcium ainsi que l'évolution des valeurs de deutérium et d'oxygène-18 suggèrent une proportion croissante d'eau géothermale, qui demeure toutefois faible (fig. 5).

\subsubsection{Exploration : identification des fuites de réservoir dans un champ géothermal}

La composition isotopique du strontium déterminée sur les fluides de Bouillante vient compléter les informations apportées par les compositions chimiques et compositions isotopiques de l'hydrogène et de l'oxygène. Sur la figure 7, la composition isotopique du strontium est portée en fonction $\mathrm{du}$ rapport des concentrations chimiques lithium sur chlore. L'asymptote à la valeur 0.7045 indique la signature isotopique en strontium de la roche encaissante composée d'andésites ou de basaltes. L'hyperbole de mélange entre le pôle de l'eau de mer et le fluide géothermal représenté par BO2 est tracée suivant les compositions de points de prélèvements qui couvrent une zone s'étendant sur une dizaine de kilomètres (de l'Ilet Pigeon situé à $5 \mathrm{~km}$ au nord de la centrale à l'Anse Thomas située à $3 \mathrm{~km}$ au sud). Ceci donne une estimation minimale de la taille du réservoir géothermal et indique notamment une zone géothermale sous-marine au nord de la baie de Bouillante non encore exploitée.

Cette hyperbole de mélange est confortée par les résultats obtenus sur les compositions isotopiques du lithium (fig. 8). Dans cette figure, les compositions isotopiques du lithium des fluides du Lamentin, en Martinique, ont également été reportées. La même hyperbole de mélange se dessine entre les pôles de l'eau de mer et du fluide géothermal de la zone géothermale du Lamentin. Néanmoins, ce fluide indique un degré d'interaction avec la roche moins avancé que celui de 
Bouillante (température du réservoir beaucoup plus faible: $90-120{ }^{\circ} \mathrm{C}$ au lieu de $260{ }^{\circ} \mathrm{C}$; Sanjuan et al., 2002). Tout ceci illustre bien le potentiel des compositions isotopiques du lithium pour tracer les processus de mélange, notamment avec l'eau de mer, isotopiquement très différente des fluides au contact avec des andésites ou des basaltes.

\subsubsection{Géothermométrie}

Les géothermomètres classiques tels que silice, Na-K, $\mathrm{Na}-\mathrm{K}-\mathrm{Ca}$ ou K-Mg appliqués sur des eaux thermales, en milieu volcanique, ne permettent pas toujours de conclure sur la température en profondeur du réservoir d'où proviennent ces eaux. On s'intéresse donc, en particulier, au géothermomètre Na-Li. Celui-ci semble dépendre également de la nature de la roche et de la quantité de $\mathrm{CO}_{2}$ présente dans le réservoir. La signature isotopique du lithium, élément apporté en solution en quantité relativement importante essentiellement par interaction avec les roches encaissantes, doit permettre de mieux comprendre le fonctionnement de ce géothermomètre, de mieux caractériser la nature minéralogique

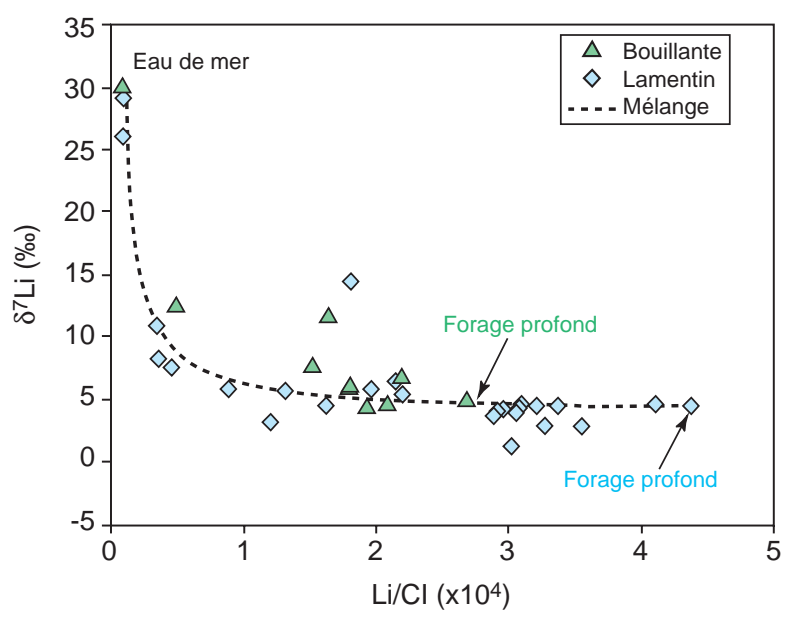

Figure 8

Variation des compositions isotopiques du lithium en fonction des rapports $\mathrm{Li} / \mathrm{Cl}$ des fluides des régions de Bouillante (Guadeloupe) et du Lamentin (Martinique).

Figure publiée initialement dans Geostandards and Geoanalytical Research (2004, vol. 28, n 1, pp. 153-159), reproduite avec la permission de l'Association scientifique pour la géologie et ses applications (ASGA) détenteur des droits.

Changes in lithium isotopic composition vs $\mathrm{Li} / \mathrm{Cl}$ ratio of the Bouillante area (Guadeloupe) and the Lamantin area (Martinique) fluids.

This figure, previously published in Geostandards and Geoanalytical Research (2004, Vol. 28, No. 1, pp. 153-159), is reproduced with the autorisation of the "Association Scientifique pour la Géologie et ses Applications" (ASGA), owner of the copyright.

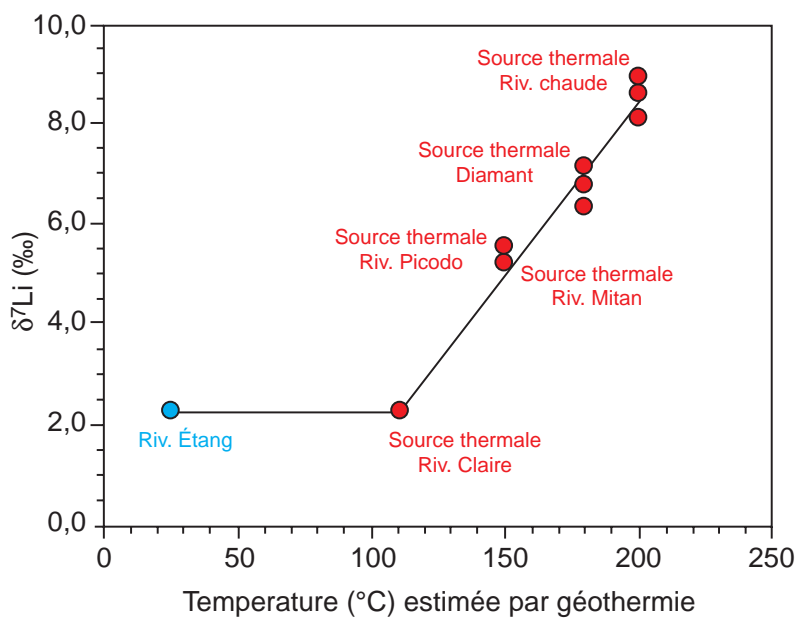

Figure 9

Variations des compositions isotopiques du lithium des eaux thermales des régions de la montagne Pelée et du Diamant (Martinique) en fonction de leur température profonde estimée par géothermométrie.

Changes in lithium isotopic composition from thermal waters of the montagne Pelée and the Diamant areas (Martinique) vs temperature and depth estimated from geothermometry.

de la roche et éventuellement, de localiser le réservoir géothermique. Les premiers travaux menés sur les eaux thermales de Bouillante sur celles des régions du Lamentin, de la montagne Pelée et du Diamant, en Martinique (Sanjuan et al., 2005), et sur celles des cirques de la Réunion sont prometteurs.

Ainsi, par exemple, une augmentation du $\delta \mathrm{Li}$ en fonction de la température profonde des eaux, établie par géothermométrie, est observée sur la figure 9 vers $100{ }^{\circ} \mathrm{C}$ pour les fluides des sources thermales des régions de la montagne Pelée et du Diamant. Ceci semble correspondre à une relation entre composition isotopique du lithium et température bien localisée, qui a permis de mettre en évidence l'existence de plusieurs réservoirs géothermiques, portés à différentes températures. Toutefois, des phénomènes d'adsorption-désorption sur les argiles formées à partir de ces roches peuvent aussi influencer le comportement du lithium et sa composition isotopique.

Des travaux expérimentaux sont actuellement en cours afin de quantifier un possible fractionnement isotopique associé à l'adsorption-désorption sur des argiles. Par ailleurs, des expériences menées en laboratoire à haute température pour des interactions eau-roche volcanique (andésite, basalte) doivent venir compléter ces résultats préliminaires concernant l'outil isotopique lithium pour la géothermie. 


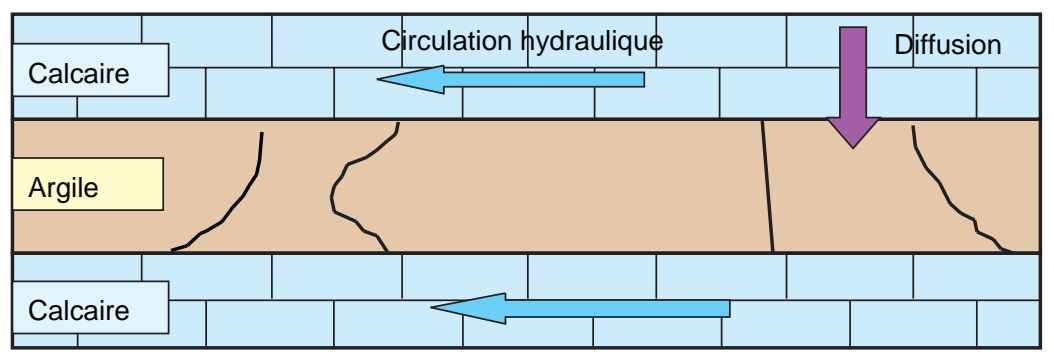

Figure 10

Schéma de profils hypothétiques de circulation d'éléments au travers d'une couche argileuse de très faible teneur en eau. Sketch of hypothetic elemental circulation profiles through a clayey layer with very low water content.

\subsection{Rôle des couches argileuses en tant que barrière naturelle}

La caractérisation à l'état zéro de sites potentiels de stockage de déchets industriels ou nucléaires met en jeu un nombre important d'outils variés. Ceux-ci vont de l'analyse chimique, isotopique et physique jusqu'à la modélisation, afin de mieux comprendre le comportement des éléments dans un milieu naturel où l'interaction entre phases liquide et solide règle le potentiel de migration de ces éléments (Lebon et Hoorelbeke, 2002). Le rôle des couches argileuses caractérisées par de faibles porosités et perméabilités est fondamental pour ce type de stockage. L'enjeu est de comprendre comment une couche argileuse a été ou sera perturbée par des phénomènes mettant en jeu un fluide majeur : l'eau. Ces études sont très spécifiques compte tenu des rapports eau/roche extrêmement faibles (teneur en eau inférieure à $5 \%$ ) et de l'implication des propriétés physicochimiques des argiles sur le comportement de l'eau.

Par ailleurs, ces couches argileuses constituent des barrières qui jouent également un rôle fondamental dans la circulation des fluides aqueux ou organiques, présents dans les bassins sédimentaires, notamment dans les séries argilogréseuses ou marno-calcaires comprenant de nombreux niveaux argileux (une telle coupe est schématisée sur la fig.10).

Une meilleure compréhension de l'organisation de la circulation des fluides dans les réservoirs, des conditions de fonctionnement de ces réservoirs et, par conséquent, de la genèse des substances fossiles qu' ils renferment permettra ainsi de diversifier et d'accroitre les sources d'approvisionnement.

Le potentiel des systèmes isotopiques Li et B est actuellement testé sur ce type de cibles en complémentarité avec d'autres systèmes $\left({ }^{18} \mathrm{O},{ }^{2} \mathrm{H},{ }^{37} \mathrm{Cl}\right.$, ), chacun d'eux étant affectés de manière différente. Pour cela, les compositions isotopiques du bore et du lithium seront caractérisées dans les eaux interstitielles et les minéraux (roche totale) de la barrière argileuse. Ces traceurs isotopiques devraient fournir des contraintes utiles à la reconstruction/modélisation des transformations passées, présentes et futures dans la couche argileuse. En particulier, il est attendu qu'ils aident à la caractérisation de la nature des fluides et des interactions qui ont eu lieu depuis le dépôt de la formation argileuse. L'identification des mécanismes de transfert des fluides et des solutés au sein de la formation (diffusion versus advection, ultrafiltration, etc.) est aussi un enjeu important (Lavastre, 2002).

Le bore et le lithium se révèlent bons traceurs de sources dans les eaux naturelles en raison de leur caractère globalement conservatif et de leur large gamme de variation isotopique (Hogan and Blum, 2003). En revanche, l'existence de fractionnements isotopiques, mal caractérisés à ce jour et liés à des réactions de dissolution/précipitation minérale ou d'adsorption/désorption sur les argiles, ne peut être ignorée (Vengosh et al., 1994 ; Bullen and McMahon, 1992). Néanmoins, l'utilisation combinée des deux traceurs isotopiques et le croisement avec les données existantes d'autres systèmes isotopiques $\left({ }^{18} \mathrm{O},{ }^{2} \mathrm{H},{ }^{37} \mathrm{Cl}\right)$ pourraient constituer un atout dans la compréhension des processus impliqués, dès lors que les fractionnement isotopiques éventuels n'affectent pas tous ces systèmes isotopiques de la même manière. $\mathrm{Cl}$ et $\mathrm{Li}$ ont souvent des comportements corrélés dans les eaux naturelles. C'est le cas notamment des eaux porales de l'Opalinus Clay, formation de Mont Terri (Suisse) (Bath and Gauschi, 2002). En revanche, le bore en solution a un comportement indépendant, plus particulièrement influencé par les conditions de $\mathrm{pH}$ (Mossadik, 1997) qui contrôlent également sa composition isotopique. Par ailleurs, les phénomènes d'adsorption/désorption affectent les isotopes du bore et du lithium en solution, le processus d'adsorption favorisant préférentiellement l'isotope léger (Zhang et al., 1998 ; Pistiner et Henderson, 2003).

L'objectif est d'établir les profils de variation verticale pour ces deux systèmes isotopiques dans les eaux porales de la couche argileuse, afin d'identifier les processus qui les ont générés.

La caractérisation des compositions isotopiques B-Li, pour un nombre suffisant d'échantillons à différentes profondeurs dans la couche cible et ses épontes, permettra de mettre en évidence l'existence (ou l'absence) de variation au sein de la série argileuse. Les profils de variation ainsi obtenus seront interprétés en terme de modèle de transfert (diffusif versus advectif) et/ou de processus (fractionnement abiotique ou 


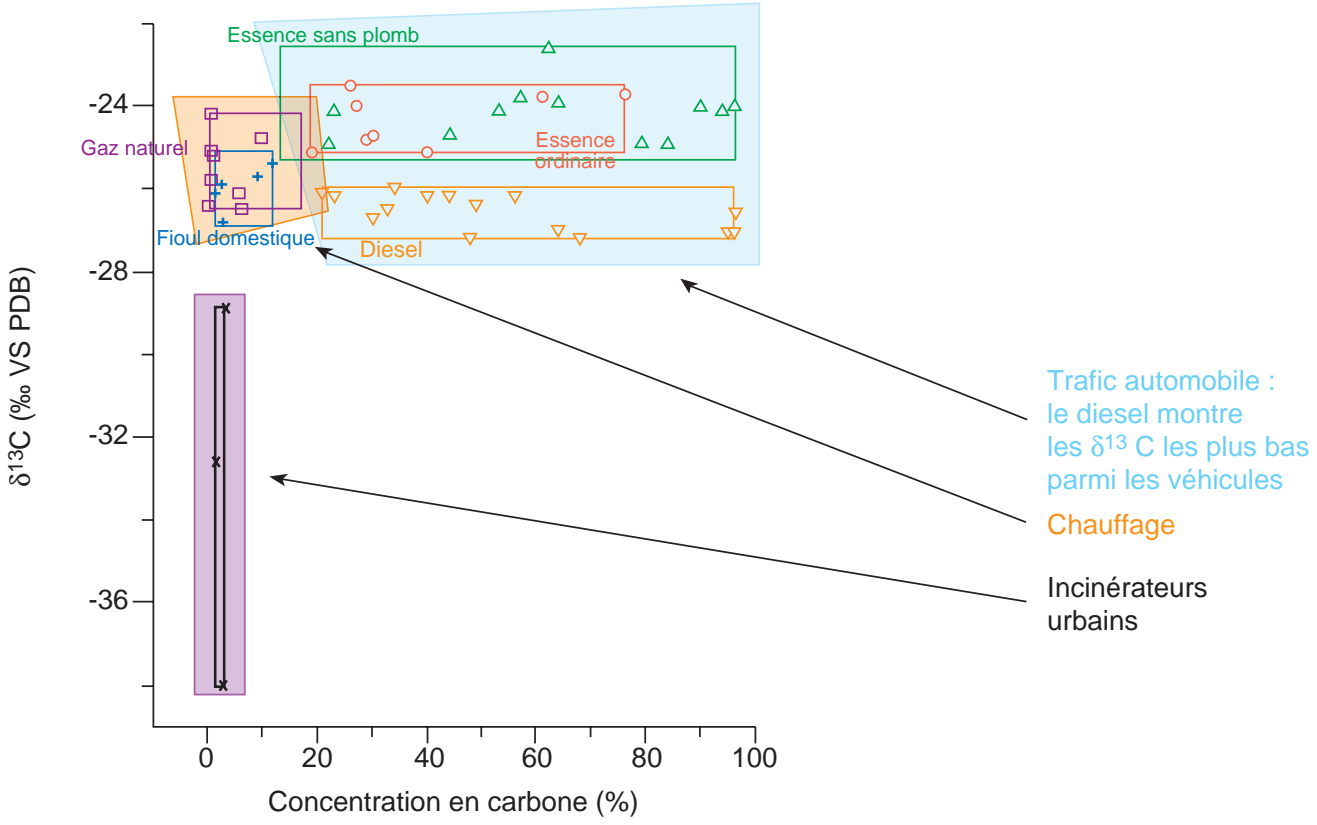

Figure 11

$\delta^{13} \mathrm{C}$ des sources de pollution dans l'atmosphère parisienne. Les incinérateurs d'ordures ménagères se distinguent très bien. En revanche, le chauffage et le trafic automobile ont des $\delta^{13} \mathrm{C}$ voisins et ne se distinguent que par la différence de concentration en carbone.

$\delta^{13} \mathrm{C}$ of the Parisian atmosphere pollution sources. Waste incinerators are well identified. On the other hand, automobile traffic and heating systems have very similar $\delta^{13} \mathrm{C}$ and can only be distinguished by their carbon concentrations.

biotique, mélanges de fluides, etc.). De plus, la variation éventuelle de la forme des profils isotopiques B-Li dans différents forages permettrait d'appréhender les variations latérales géographiques des processus associés.

Les compositions isotopiques du $\mathrm{B}$ et $\mathrm{Li}$ renseigneront $a$ minima sur l'origine des eaux porales. Elles permettront notamment de vérifier l'existence ou non du signal marin résiduel des conditions de dépôt initiales.

\subsection{Protection de l'environnement et développement durable}

Les préoccupations des pouvoirs publics en matière de protection de l'environnement induisent des normes antipollution de plus en plus sévères. La pollution atmosphérique est actuellement une préoccupation majeure et l'utilisation de l'outil isotopique en tant que traceur se développe rapidement. Le potentiel de cet outil est d'identifier les sources de pollution et de semi-quantifier leur contribution. Dans ce cas, l'objectif peut être de savoir sur quelle source jouer quand l'apport est trop important par rapport à une limite réglementaire. Une illustration en est donnée par une étude multiisotopique de la pollution atmosphérique de Paris (Widory et al., 2004a et 2004b).

Du fait de leurs concentrations relativement élevées en milieu urbain (de 10 à plus de $50 \mu \mathrm{g} \cdot \mathrm{m}^{-3}$ ), les particules sont une forme de pollution atmosphérique ayant une incidence sur la santé publique. La réduction de cette pollution implique d'en connaître les sources prépondérantes et dans cette recherche, la seule utilisation des paramètres de caractérisation minérale et de caractérisation chimique s'est avérée très souvent insuffisante. Les principales sources de particules atmosphériques de l'agglomération parisienne sont la circulation automobile et les sources fixes de combustion (chauffages urbains et incinérateurs d'ordures ménagères).

Les résultats sont reportés dans les figures 11 à 14 . Ils montrent que l'étude multi-isotopique du carbone, de l'azote, $\mathrm{du}$ strontium et du plomb permet de discriminer de manière non équivoque les différentes sources d'émission de PM10 (matière particulaire de diamètre inférieur à $10 \mu \mathrm{m}$ ) dans l'atmosphère parisienne (Widory et al., 2004a, 2004b).

Il est alors possible, à partir de la caractérisation multiisotopique d'un prélèvement effectué en air ambiant, d'apporter des informations pertinentes quant à l'origine des particules échantillonnées.

Les mesures de $\delta^{13} \mathrm{C}$, de ${ }^{87} \mathrm{Sr} /{ }^{86} \mathrm{Sr}$ et des rapports isotopiques du plomb permettent de tracer directement l'origine des PM10, et de plus, de discriminer une origine différente pour les phases organique $(\mathrm{C}, \mathrm{N})$ et inorganique $(\mathrm{Pb}, \mathrm{Sr})$ le cas échéant. Les mesures de $\delta^{15} \mathrm{~N}$ apportent en plus une information sur l'origine des oxydes d'azote (NOx) lors de leur changement de phase dans les phénomènes 
post-combustion (i.e. passage de l'état gazeux à l'état solide et agrégation sur les particules préexistantes).

Cette étude sera étendue à d'autres systèmes isotopiques et les plus discriminants seront sélectionnés.

\section{AUTRES DÉVELOPPEMENTS EN COURS}

\subsection{Fer}

Ces dernières années, l'existence de fractionnements significatifs des isotopes du $\mathrm{Fe}\left(\delta^{56} \mathrm{Fe}\right)$ a été mis en évidence (Zhu et al., 2002 ; Anbar, 2004) dans les systèmes naturels, et la caractérisation des mécanismes de fractionnement fait l'objet de nombreux travaux scientifiques en cours. Ces études documentent la variabilité naturelle du $\delta^{56} \mathrm{Fe}$ et des fractionnements isotopiques associés à des processus chimiques, géochimiques et biologiques. À basse température, la spéciation du fer en solution et les phénomènes d'oxydoréduction influencent fortement la composition isotopique du $\mathrm{Fe}$ dissous. Par ailleurs, des fractionnements importants sont générés par l'activité de microorganismes ferriréducteurs ou ferrioxydants, qui semblent incorporer les isotopes légers du Fe de façon préférentielle. Un fractionnement isotopique significatif (jusqu'à $2 \%$ environ) a été mis en évidence lors de la réduction/oxydation du fer en présence de bactéries (Motelica-Heino et al., 2004a, 2004b). Il apparaît donc que les variations isotopiques du $\mathrm{Fe}$ peuvent apporter un éclairage sur les processus d'oxydoréduction et sur l'activité bactérienne dans les eaux de surface et subsurface. Le $\delta^{56} \mathrm{Fe}$ constituerait non seulement un traceur des conditions d'oxydoréduction du milieu considéré, mais aussi un marqueur potentiel, qualitatif et quantitatif des interactions géosphère/biosphère et des processus microbiens.

\subsection{Cadmium}

Ce métal lourd toxique est un élément ultra-trace, c'est-à-dire que le facteur limitant la précision de la mesure est la quantité de $\mathrm{Cd}$ disponible par échantillon, ce qui nécéssite une chimie préparatoire fine (Innocent, 2004a, 2004b). En raison des très faibles fractionnements isotopiques attendus, l'utilisation d'un double marqueur isotopique (spike) est, en théorie, de loin préférable à la technique classique d'encadrement par des standards pour la mesure en ICP-MS-MC, cette technique étant de toute façon indispensable pour la mesure en TIMS. Toutefois, elle ne sera réellement performante que dans la mesure où les quantités de $\mathrm{Cd}$ disponibles permettront d'obtenir des signaux assez intenses (ce qui n'est pas toujours possible). Les premiers essais ont été effectués avec un double spike ${ }^{111} \mathrm{Cd}^{1}{ }^{116} \mathrm{Cd}$ (Innocent, 2004a). Par la suite, un nouveau double spike ${ }^{110} \mathrm{Cd}-{ }^{116} \mathrm{Cd}$, plus adéquat, a été calibré et pourra servir pour les analyses en TIMS et en ICPMS-MC (Innocent, 2004b).
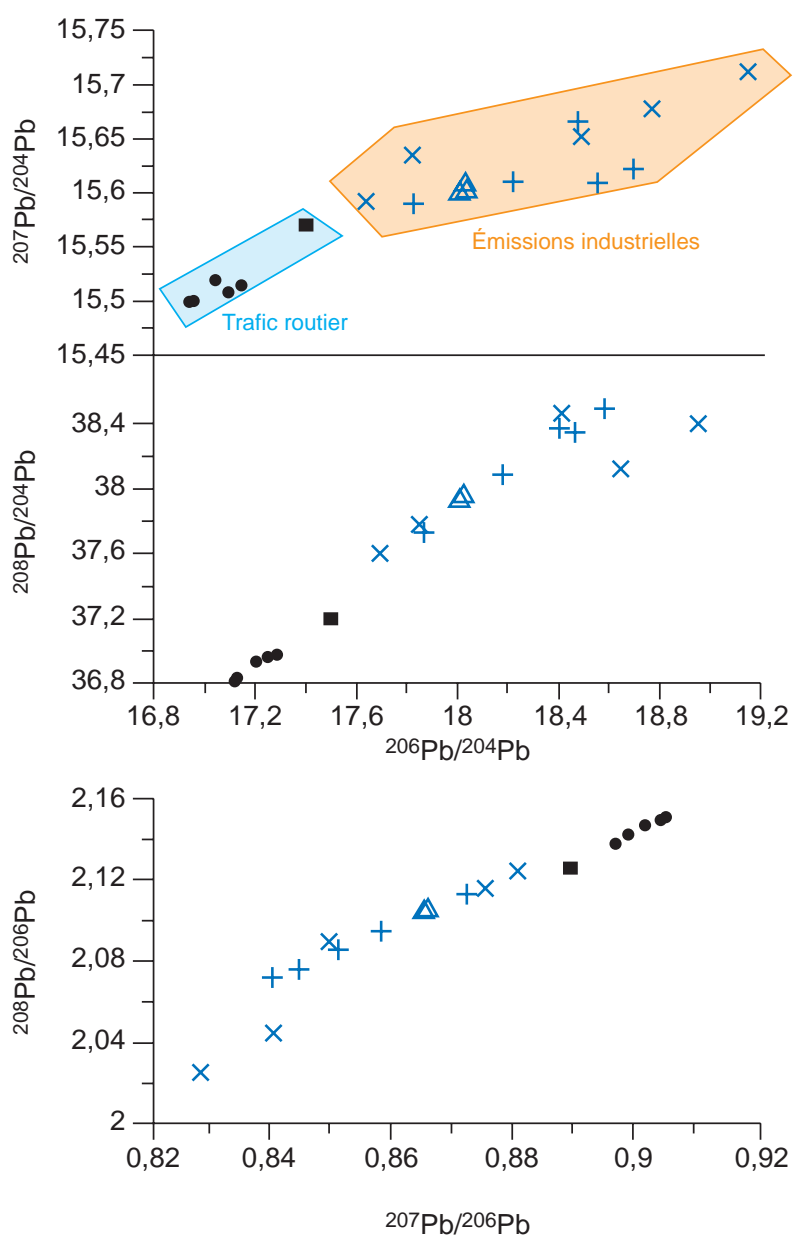

Figure 12

Compositions isotopiques du plomb des sources de pollution dans l'atmosphère parisienne. Le trafic routier se distingue très bien des émissions industrielles (chauffage et incinérateurs).

-: Prélèvements de pots d'échappement de véhicules.

$\Delta$ : Incinérateurs urbains de Paris (SYCTOM).

+ : Compagnie de chauffage anonyme (combustibles: gaz naturel, charbon et mazout).

$\times$ : Compagnie Parisienne de Chauffage Urbain (CPCU) (combustibles : gaz et mazout).

Lead isotopic compositions of the parisian atmosphere pollution sources. Road traffic distinguishes very well from industrial emissions (heating systems and incinerators).

•: Sampling of vehicle exhaust.

$\Delta:$ Urban incinerators of Paris (SYCTOM)

+: Anonymous heating company (fuels: natural gas, coal and fuel oil)

$\times$ : Urban Heating Company of Paris (CPCU) (fuels: gas and fuel oil)

La mise en évidence et la quantification des pollutions ponctuelles et diffuses en milieu urbain, mais aussi rural, résultant de divers processus anthropiques (épandages d'engrais, incinérations, raffinages, procédés industriels divers, relargage de microdéchets de produits manufactrurés) constituent l'objectif premier de l'utilisation des isotopes de $\mathrm{Cd}$. 


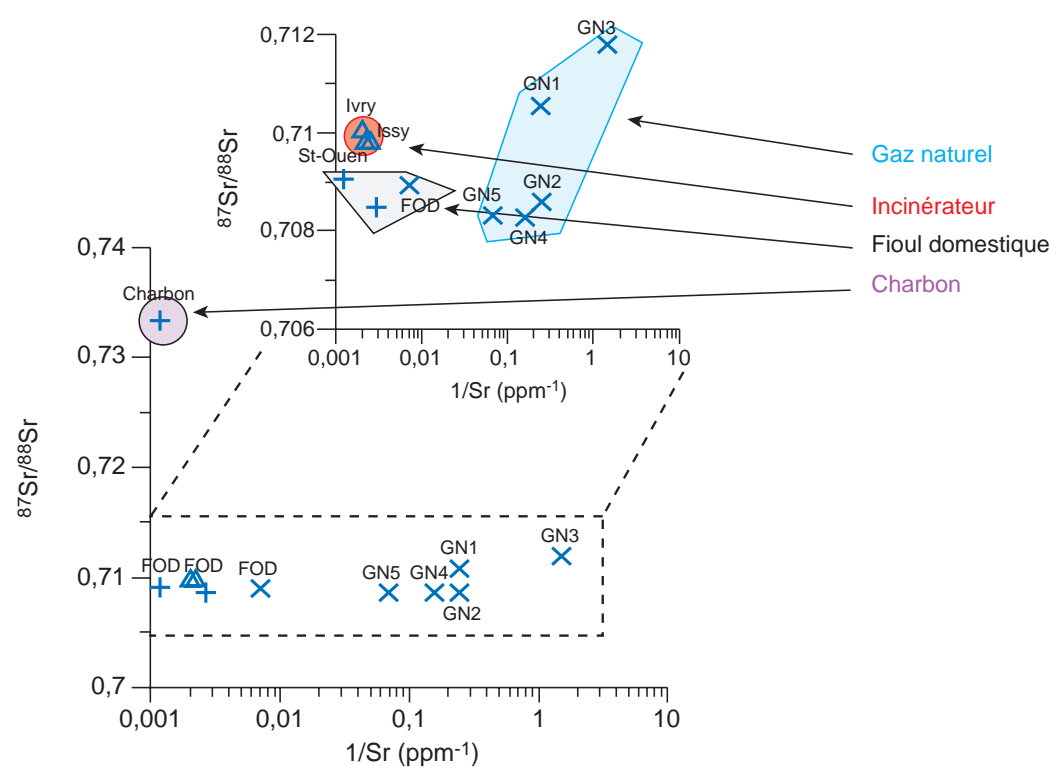

Figure 13

Compositions isotopiques du strontium des sources de pollution (Paris). Le strontium discrimine le gaz naturel, le fioul domestique, le charbon et les incinérateurs d'ordures ménagères. Les concentrations en strontium dans les émissions du trafic automobile sont inférieures aux limites de détection.

Strontium isotopic compositions of the pollution sources (Paris). Strontium isotopes distinguish natural gas, domestic oil, coal and household waste incinerators. Strontium isotope concentrations from automobile traffic samples are bellow detection limit.

\subsection{Bore}

L'analyse isotopique du bore en TIMS, si elle est parfaitement maîtrisée, présente l'inconvénient de nécessiter une grande quantité de bore (chargement dans le TIMS de l'ordre du $\mu \mathrm{g}$, traitement chimique entre 5 et $10 \mu \mathrm{g}$ ). L'accès à des échantillons très pauvres (concentration inférieure au $\mathrm{ppb}$ ) demanderait de tels volumes d'échantillon, que ce type d'analyse n'est pas actuellement envisagé. En revanche, l'utilisation de l'ICP-MS-MC est une excellente alternative puisque les tests effectués montrent que $100 \mathrm{ng}$ de $\mathrm{B}$ total sont largement suffisants (analyse sur une solution à $50 \mathrm{ppb}$ ). Le facteur limitant reste la nécessité d'effectuer une séparation chimique préalable pour éliminer les effets de matrice, la contamination étant actuellement de l'ordre de 1-2 ng, soit 1 à $2 \%$ du bore traité. La reproductibilité est de l'ordre de $0,25 \%$ (Guerrot et al., 2003). La validation de cette procédure est en cours, et l'on devrait à terme avoir accès à l'analyse isotopique du bore d'eaux très faiblement concentrées ou de micro-échantillons.

\subsection{Plomb}

Les analyses isotopiques du plomb, bien qu'effectuées en routine sur TIMS, restent délicates pour des échantillons pauvres en plomb (eau de surface, eau de pluie), ou pour des échantillons en faible quantité (poussières urbaines). L'ICPMS-MC est donc utilisé à cette fin pour déterminer les sources naturelles ou anthropogéniques d'échantillons environnementaux, ceci sans avoir à effectuer des séparations chimiques délicates et susceptibles de polluer l'échantillon. Des tests très convaincants montrent qu'une charge cationique élevée n'a pas d'incidence sur la précision et la justesse des rapports isotopiques du $\mathrm{Pb}$ : jusqu'à un équivalent de $400 \mathrm{mg} / \mathrm{l}$ (soit une solution à $40 \mathrm{~g} / \mathrm{l}$ diluée 100 fois). Jusqu'à ces conditions limites et pour une teneur en $\mathrm{Pb}$ minimale de $10 \mathrm{ppb}$, il est possible d'assurer des erreurs absolues (à $2 \mathrm{~s}$ ) de $0,1 \%\left({ }^{206} \mathrm{~Pb} /{ }^{204} \mathrm{~Pb},{ }^{207} \mathrm{~Pb} /{ }^{204} \mathrm{~Pb}\right.$ et $\left.{ }^{208} \mathrm{~Pb} /{ }^{204} \mathrm{~Pb}\right)$ et de $0,01 \%$ ${ }^{207} \mathrm{~Pb} /{ }^{206} \mathrm{~Pb}$ et $\left.{ }^{208} \mathrm{~Pb} / 206 \mathrm{~Pb}\right)$.

\subsection{Perspectives de couplage ICP-MS-MC et HPLC}

Peut-on rêver de mesurer les compositions isotopiques d'un élément et sa spéciation en simultané ? A priori, oui. Un couplage entre l'ICP-MS-MC et, par exemple, un appareil de chromatographie liquide, permettrait de mesurer la composition isotopique de chaque forme chimique d'un même élément. Dans ce cas, la compréhension du comportement physicochimique d'un élément, à l'échelle de chaque type de molécule, est envisageable. Les applications concrètes concerneraient des problèmes d'environnement (eaux naturelles, résiduaires, sols), mais également des questions de santé publique, où un traçage de chaque molécule, au sein même des processus métaboliques humains, pourrait être abordé (cas du saturnisme, par exemple). 


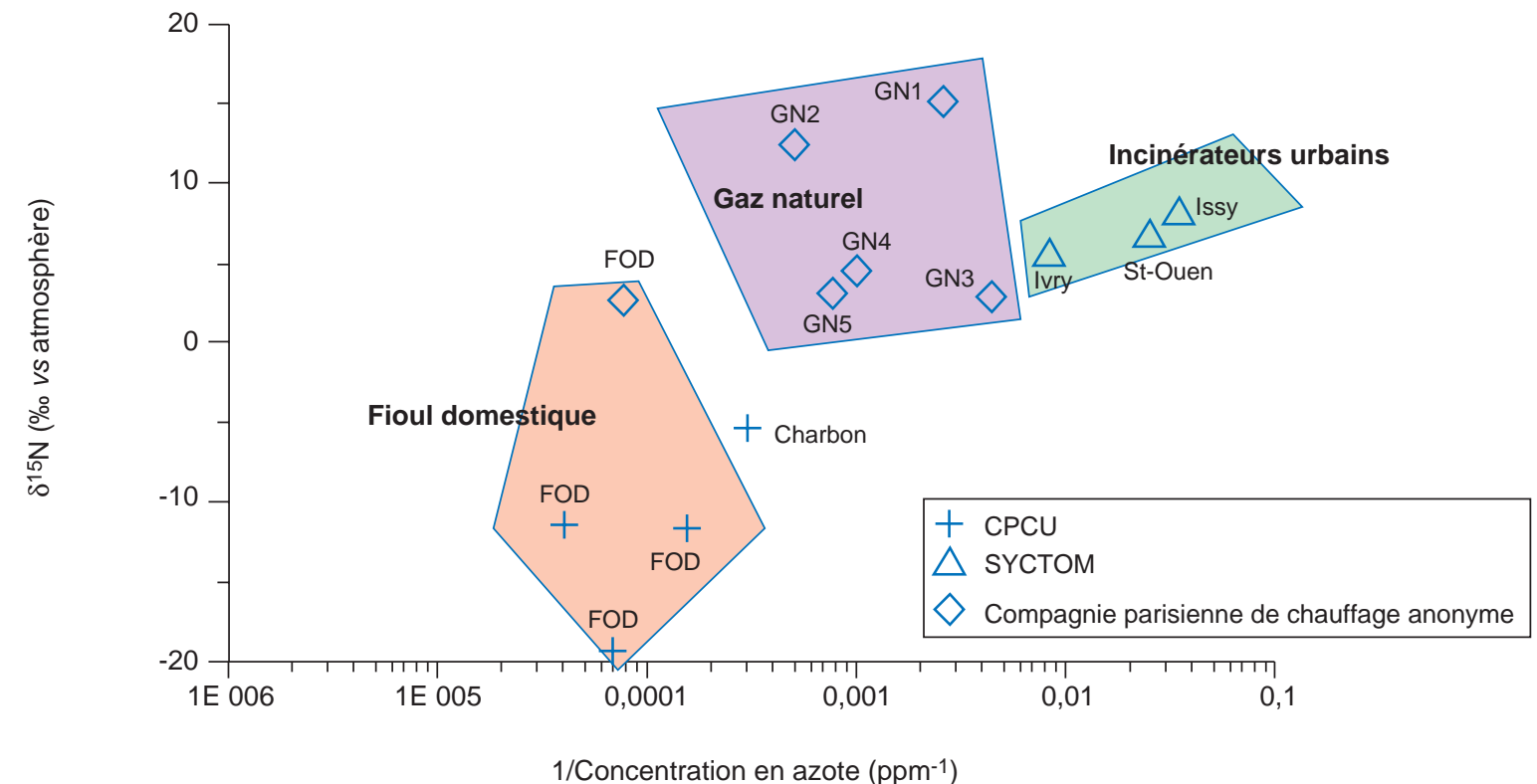

Figure 14

Compositions isotopiques de l'azote des sources de pollution (Paris). Les $\delta^{15} \mathrm{~N}$ permettent de discriminer les différentes sources fixes.

Nitrogen isotopic compositions of the pollution sources (Paris). $\delta^{15} \mathrm{~N}$ permits to distinguish the different ground sources.

\section{CONCLUSION}

La communauté des isotopistes a assisté ces dernières années à une avancée technologique qui rend les systèmes isotopiques classiques plus accessibles et qui ouvre de nouveaux champs d'investigation, avec l'accès à de nouveaux systèmes isotopiques.

Dans la démarche logique d'une recherche d'efficacité de la caractérisation minérale, des mesures physiques et chimiques sont mises en jeu avant l'utilisation des traceurs isotopiques. Lorsque des questions subsistent, les études multitraceurs isotopiques sont introduites et se révèlent être un outil d'une grande puissance en appui à la compréhension des systèmes de mélange à plusieurs pôles.

\section{RÉFÉRENCES}

Anbar, A.D. (2004) Iron stable isotopes: beyond biosignatures. Earth and Planetary Science Letters, 217, 223-236.

Bath, A. et Gauschi, A. (2002) Evolution of pore water in the Opalinus Clay at Mont Terri, Switzerland. Clays in Natural and Engineered Barriers for Radioactive Waste Confinement, Int. Meeting, 9-12 déc. Reims, France.

Bullen, T.D. et McMahon, P.E. (1992) Evolution of ${ }^{87} \mathrm{Sr} /{ }^{86} \mathrm{Sr}$ and $\mathrm{d}^{7} \mathrm{Li}$ in groundwater from the Black creek aquifer: confirmation of a model for the origin of $\mathrm{Na}^{+}-\mathrm{HCO}^{-}$waters in a clastic aquifer. $\operatorname{EOS} 73, \mathrm{~S} 139$.

Chan, L.H. (1987) Lithium isotope analysis by thermal ionization mass spectrometry of lithium tetraborate. Analytical Chemistry, $59,2662-2665$.
Coleman, M. Eggenkamp, H. et Aranyossy, J.F. (2001) Chlorine stable isotope characterisation of solute transport in mudrocks. Actes des Journées Scientifiques Andra, 7-9 déc. 1999, Nancy, France, 155-175.

Gaucher, E., Robelin, C., Matray, J.M., Négrel, G., Gros, Y., Vinsot, A., Rebours, H., Cassagnabère, A. et Bouchet, A. (sous presse). Andra underground research laboratory: interpretation of the mineralogical and geochemical data acquired in the CallovoOxfodian formation by investigative drilling. Applied Clay Science, sous presse.

Guerrot, C., Robert, M. et Millot, R. (2003) L'analyse isotopique du bore par MC-ICP-MS. Spectr'Atom 2003, Pau, 9-11 avril.

Hogan, J.F. et Blum, J.D. (2003) Boron and lithium isotopes as groundwater tracers: a study at the Fresh Kills Landfill, Staten Island, New York, USA. Applied Geochem., 18, 615-627.

Innocent, C. (2004a) Isotopic composition of Cd in terrestrial materials: new insights from a high-precision, double spike analytical method. Environmental Chemistry, Springer-Verlag, sous presse.

Innocent, C. (2004b) Cd isotopic composition measured by plasma source mass spectrometry in natural and anthropogenic materials. Abstract, $3^{e}$ journées SFIS, Paris, sept.

James R.H. et Palmer, M.R. (2000) The lithium isotope composition of international rock standards. Chemical Geology, 166, 319-326.

Lavastre, V. (2002) Événements sédimentaires, diagénétiques et post-diagénétiques dans la formation argileuse du CallovoOxfordien (bassin de Paris, France) : enregistrement isotopique des minéraux et de l'eau porale. Thèse de l'université Paris VII.

Lebon, P. et Hoorelbeke, J.M. (2002) Clays in radioactive waste containment: expected role and strategy for data acquisition. Int. Meeting on Clays in Natural and Engineered Barriers for Radioactive Waste Confinement, Abs., Reims, 9-12 déc., 5-6. 
Millot, R., Guerrot, C. et Girard, J.P. (2003) Analyse des isotopes du lithium par ICP-MS-MC. Spectr'Atom 2003, Pau, 9-11 avril.

Millot, R., Guerrot, C. et Vigier, N. (2004) Accurate and highprecision measurement of lithium isotopes in two reference materials by MC-ICP-MS. Geostandards and Geoanalytical Research, 28, 153-159.

Moriguti, T. et Nakamura, E. (1998) High-yield lithium separation and the precise isotopic analysis for natural rock and aqueous sample. Chemical Geology, 145, 91-104.

Mossadik, H. (1997) Les isotopes du bore, traceurs naturels dans les eaux : mise au point de l'analyse en spectrométrie de masse à source solide et applications à différents environnements. Thèse de l'université d'Orléans.

Motelica-Heino, M., Hughet, L., Diot, G. et Garrido, F. (2004a) Iron isotopic fractionation during the dissolutive reduction of synthetic iron oxides by micro-organisms. Winter Conference on Plasma Spectrochemistry, Fort Lauderdale, États-Unis.

Motelica-Heino, M., Casiot, C. et Elbaz, F. (2004b) Iron isotopic fractionation during microbial and abiotic oxidation. Goldsmidt Conference, Copenhague, Danemark.

Nishio, Y. et Nakai, S. (2002) Accurate and precise lithium isotopic determinations of igneous rock samples using multicollector inductively coupled plasma mass spectrometry. Analytica Chimica Acta, 456, 271-281.

Pistiner, J.S. et Henderson, G.M. (2003) Lithium isotope fractionation during continental weathering processes. Earth and Planetary Science Letters, 214, 327-339.

Sanjuan, B., Lasne, E. et Brach, M. (2000) Bouillante geothermal field (Guadeloupe, West Indies): geochemical monitoring during a thermal stimulation operation. Proceedings, $25^{\text {th }}$ Workshop on Geothermal Reservoir Engineering, université de Stanford, Californie, États-Unis, 24-26 janv., 215-222.

Sanjuan, B., Lasne, E. et Brach, M. (2001) Bouillante geothermal fluid: mixing and water/rock interaction processes at $250^{\circ} \mathrm{C}$. Proceedings, 10 th Water-Rock Interaction (WRI-10), éd. Rosa Cidu, A.A. Balkema Publishers, Cagliari, Italie, 10-15 juin, 2, 911-914.
Sanjuan, B., Traineau, H., Genter, A., Correia, H., Brach, M. et Degouy, M. (2002) Geochemical investigations during a new geothermal exploration phase in the Lamentin Plain (Martinique, French West Indies). Proceedings, $27^{\text {th }}$ Workshop on Geothermal Reservoir Engineering, université de Stanford, Californie, ÉtatsUnis, 28-30 janv., 198-205.

Sanjuan, B., Millot, R., Brach, M., Foucher, J.C., Roig, J.Y. et Baltassat, J.M. (2005) Geothermal exploration in the Mount Pelée volcano-Morne Rouge and Diamant areas (Martinique, West French Indies): geochemical data. Proceedings World Geothermal Congress, avril, Antalya, Turquie.

Tonnarini, S., Pennisis, M. et Leeman, W. (1997) Precise boron isotopic analysis of complete silicate (rock) samples using alkali carbonate fusion and ion-exchange separation. Chem. Geol, 142, 129-137.

Vengosh, A., Heumann, K.G., Juraske, S. et Kasher, R. (1994) Boron isotope application for tracing sources of contamination in groundwater. Envir. Sci. Technol., 28, 1968-1974.

Widory, D., Roy, S., Le Moullec, Y., Goupil, G., Cocherie, A., Guerrot, C. et al. (2004a) The origin of atmospheric particles in Paris: a view through carbon and lead isotopes. Atmospheric Environment, 38, 953-961.

Widory, D., Fiani, E., Le Moullec, Y., Gruson, S. et Gayrard, O. (2004b) Contribution des sources fixes à la pollution particulaire à Paris : apport des isotopes. Rapport BRGM.

You, C.F. et Chan, L.H. (1996) Precise determination of lithium isotopic composition in low concentration natural samples. Geochimica Cosmochimica Acta, 60, 909-915.

Zhang, L., Chan, L.H., et Gieskes, J.M. (1998) Lithium isotope geochemistry of pore waters from Ocean Drilling Progam sites 918 and 919, Irminger Basin. Geochimica Cosmochimica Acta, $62,2437-2450$

Zhu, X.K., Guo, Y., Williams, R.P.J., O’Nions, R.K., Matthews, A., Belshaw, N.S., Canters, G.W., de Wall, E.C., Weser, U., Burgess, B.K. et Salvato, B. (2002) Mass fractionation processes of transition metal isotopes, Earth Planet. Sci. Lett., 200, 47-62.

Manuscrit définitif reçu en février 2005 